\title{
MicroRNA-221 inhibits the transition of endothelial progenitor cells to mesenchymal cells via the PTEN/Fox03a signaling pathway
}

\author{
${ }^{*}$ En Zhou ${ }^{1, C-E}$, ${ }^{*}$ Yinghua Zou ${ }^{2, C-E}$, ${ }^{*}$ Chengyu Ma0 ${ }^{1, C-E},{ }^{*}$ Dongjiu Li ${ }^{1, C-E}$, Changqian Wang ${ }^{1, A, F}$, Zongqi Zhang ${ }^{1, A, E, F}$ \\ ${ }^{1}$ Department of Cardiology, Shanghai Ninth People's Hospital, School of Medicine, Shanghai Jiao Tong University, China \\ ${ }^{2}$ Department of Anesthesiology, Shuguang Hospital, Shanghai University of Traditional Chinese Medicine, China \\ A - research concept and design; $\mathrm{B}$ - collection and/or assembly of data; C - data analysis and interpretation; \\ $D$ - writing the article; $E$ - critical revision of the article; $F$ - final approval of the article
}

\section{Address for correspondence}

Zongqi Zhang

E-mail: zqzhangdocto@@126.com

\section{Funding sources}

National Natural Science Foundation of China (grant NNo. 81200206 to ZQ Zhang); Scientific Research and Innovation Project of Shanghai Education Commission (grant No. 12 YZO45 to ZQ Zhang); Shanghai Committee of Science and Technology, China (grant No. 18411950500); National Natural Science Foundation of China (grant №. 81870264).

Conflict of interest None declared

*En Zhou, Yinghua Zou, Chengyu Mao and Dongjiu Li contributed equally to this study.

Received on March 9, 2021

Reviewed on May 16, 2021

Accepted on August 19, 2021

Published online on 0ctober 5, 2021

Cite as

Zhou E, Zou Y, Mao C, Li D, Wang C, Zhang Z. MicroRNA-221 inhibits the transition of endothelial progenitor cells to mesenchymal cells via the PTEN/Fox03a signaling pathway. Adv Clin Exp Med. 2021;30(12):1263-1270. doi:10.17219/acem/141446

DOI

10.17219/acem/141446

Copyright

Copyright by Author(s)

This is an article distributed under the terms of the

Creative Commons Attribution 3.0 Unported (CC BY 3.0)

(https://creativecommons.org/licenses/by/3.0/)

\begin{abstract}
Background. Coronary heart disease is one of the most common cardiovascular diseases worldwide and is often associated with vascular endothelial injury. Endothelial-mesenchymal transition (EndMT) is an important process in vascular endothelial injury.

Objectives. This study investigated the function of miR-221 in the EndMT process of endothelial progenitor cells (EPCS).

Materials and methods. Transforming growth factor beta (TGF- $\beta 1$ ) was used to induce EndMT in EPCS, and SM22a expression was detected using immunocytochemistry. Western blot was used to detect alpha smooth muscle actin (aSMA) expression, and miR-221 function was evaluated using inhibitors or mimics of the miR-221 sequences that were transfected into EPCs. Reverse transcription polymerase chain reaction (RT-PCR) was used to detect the expression of miR-221 and western blot was used to detect the expression of aSMA, myocardin, phosphatase and tensin homolog (PTEN), p-Fox03a, and Fox03a in EPCs. Finally, the expression of the miR-221 target genes was determined using RT-PCR.

Results. The expression of SM22a and aSMA increased in EPCs treated with TGF- $\beta 1$, while the expression of miR-221 was decreased in EPC on the $5^{\text {th }}$ day, when compared with the control. The expression of SM22a increased after inhibiting miR-221 in EPCs treated with TGF- $\beta 1$ and this was reversed by the overexpression of miR-221. The expression of aSMA and myocardin was significantly increased after inhibiting miR-221 in EPCs treated with TGF- $\beta 1$ and decreased in EPCs overexpressing miR-221. Conversely, PTEN was increased in TGF- $\beta 1$-treated EPC $s$ and decreased following the overexpression of miR-221. The decrease in phosphorylated-Fox03a expression in EPCs was accompanied by an increase in aSMA expression, which was reversed in the presence of miR-221 mimics. This effect was nearly abolished following the addition of PTEN CDNA.
\end{abstract}

Conclusions. The overexpression of miR-221 inhibits EndMT in EPCs, possibly by interacting with PTEN to regulate Fox03a signaling, to facilitate the repair of the endothelium by EPCS.

Key words: EPC, miR-221, EndMT, PTEN/Fox03a 


\section{Background}

Coronary heart disease is one of the most common cardiovascular diseases worldwide and is caused by vascular endothelial injury. It has been demonstrated that once the vascular endothelium is damaged, the endothelial progenitor cells (EPCs) migrate from the bone marrow $(\mathrm{BM})$ to the ischemic tissue and differentiate into mature vascular endothelial cells in an attempt to repair the injured site. ${ }^{1}$ However, accumulating evidence suggests that EPCs contribute to intimal hyperplasia during this process. For example, BM-derived EPCs have been shown to augment intimal hyperplasia by migrating into the intima of balloon-injured carotid arteries, ${ }^{2}$ and our previous study showed that BM-derived EPCs can transdifferentiate into smooth muscle lineage cells that are positive for alpha smooth muscle actin ( $\alpha$ SMA), producing an endothelialto-mesenchymal transition (EndMT). ${ }^{3}$ These results suggest that the EndMT capacity of EPCs may contribute to intimal hyperplasia. This is further supported by evidence that EndMT increases the thickness of the intimal layer in pulmonary arteries. ${ }^{4}$ However, the mechanisms underlying the EndMT process in EPCs have not yet been fully elucidated.

MicroRNAs (miRs) are short (only 21-23 nucleotides), highly conserved, non-coding RNA sequences that bind to the 3'-untranslated region of a variety of mRNA targets and function to regulate gene expression at the posttranscriptional level by inhibiting the translation or promoting mRNA degradation. ${ }^{5,6}$ Previous microarray-based expression profiles have shown that several miRs are expressed in EPCs, including miR-126, miR-21, miR-27a, and miR-221. ${ }^{7,8}$ Furthermore, some studies have shown that both miR-21 and miR-127 are involved in mature endothelial cell EndMT that can be induced by TGF- $\beta 1$ and TGF- $\beta 2 .^{8,9}$ In addition, miR-221, an endothelial-specific miR, has been shown to exert anti-angiogenic, anti-inflammatory and pro-apoptotic effects by targeting both ZEB2 and Ets-1. ${ }^{10,11}$ Finally, miR-221 maintains the angiogenic capacity of EPCs by inhibiting p27 expression. ${ }^{12}$ However, few studies have described the role of miR-221 in the EndMT process of EPCs.

Previous investigations have confirmed that phosphatase and tensin homolog (PTEN) is a direct target of miR-221. ${ }^{13}$ In vascular endothelial cells, downregulation of miR-221 decreased cell viability, migration and invasion, while all of these effects were reversed in response to the overexpression of PTEN. ${ }^{13}$ Furthermore, recent studies have shown that a decrease in PTEN activates the phosphorylation of FoxO3a. ${ }^{14,15}$ In our previous study, we showed that phosphorylated FoxO3a plays an inhibitory role in EndMT by inactivating the Smad3/4 pathway in the nucleus of EPCs. ${ }^{16}$ Given this, we hypothesized that the activation of the miR-221/PTEN/FoxO3a pathway may inhibit EndMT in EPCs.

\section{Objectives}

This study was designed to determine the function of miR-221 in the EndMT process of EPCs and to explore the potential signaling pathways underlying these functions.

\section{Materials and methods}

\section{Ethical approval}

All animal experiments were approved by the Animal Research Ethics Committee of the No. 9 People's Hospital affiliated with the Shanghai Jiao Tong University School of Medicine, Shanghai, China. All animals were sacrificed using bilateral thoracotomy during anesthesia.

\section{Cell isolation and culture}

Bone marrow-derived EPCs were isolated from male Sprague Dawley rats, as described previously. ${ }^{2}$ FicollIsopaque Plus (Histopaque-1077; Sigma-Aldrich, St. Louis, USA) density gradient centrifugation was used to isolate mononuclear cells from rat bone marrow. The cells were cultured in endothelial cell growth medium (EGM2; Lonza, Basel, Switzerland) consisting of endothelial cell basal medium-2 (EBM-2), supplemented with $20 \%$ fetal bovine serum (FBS). Cells were then cultured on a fibronectin-coated dish at $37^{\circ} \mathrm{C}$ in a $5 \% \mathrm{CO}_{2}$ incubator. The medium was removed after 4-7 days, and cells were gently washed with phosphate-buffered solution (PBS). The attached cells were then used for further experiments. The CD31 fluorescence staining was then performed as previously described ${ }^{7}$ to identify vascular endothelial cells.

\section{Inducing EndMT in EPCs}

Endothelial progenitor cell EndMT was induced as previously described. ${ }^{17}$ Briefly, the cells were seeded on culture plates in Ham's F-12 media containing 5\% FBS, and then treated with TGF- $\beta 1$ ( $5 \mathrm{ng} / \mathrm{mL})$ for 7 days.

\section{Immunocytochemistry}

The EPCs were cultured on cell slides and then rinsed with PBS, before being fixed with $4 \%$ paraformaldehyde phosphate buffer for $15 \mathrm{~min}$ at room temperature. Slides were then blocked for $2 \mathrm{~h}$ in PBS containing 5\% normal goat serum and $2 \%$ bovine serum albumin (BSA), and incubated with monoclonal antibodies (diluted 1:500) against SM22 $\alpha$ (Cat. No. SAB1400272; Sigma-Aldrich) overnight at $4^{\circ} \mathrm{C}$. Cells were washed 5 times in PBS and incubated with AlexaFluor ${ }^{\circledR}$ 594-conjugated goat anti-mouse/rabbit antibody diluted 1:500 (Santa Cruz Biotechnology, Dallas, USA) for $1 \mathrm{~h}$ at room temperature. The mean fluorescence intensity of SM22 $\alpha$ was 
evaluated using ImageJ software (RRID:SCR_003070; National Institutes of Health (NIH), Bethesda, USA).

\section{Real-time PCR}

Total RNA was isolated from EPCs using TRIzol reagent (Cat. No. 10296028, Lot No. 79348502; Life Technologies, Carlsbad, USA), as described in the manufacturer's instructions. Extracted RNA was then reverse transcribed to cDNA using a Thermo Cycler (Applied Biosystems, Waltham, USA) and real-time polymerase chain reaction (RT-PCR) was performed using a real-time PVR machine (LightCycler 480 II; Roche, Basel, Switzerland). The mRNA was quantified using a TaKaRa reverse transcription assay (Cat. No. R0037A; TaKaRa, Tokyo, Japan) and SYGRII (Cat. No. RR820A; TaKaRa). Gene-specific primers were synthesized by Sangon Biotech (Shanghai, China) and mRNA levels were quantified using the $2^{-\Delta \Delta c t}$ method (Table 1 ).

\section{Transfection}

The miR-221 mimics and inhibitors were synthesized using the Qiagen (Hilden, Germany) reagents and the miR-221 mimic sequence was set as 5'-AGCUACAUUGUCUGCUGGGUUUC-3'. The gene ID of PTEN in National Center for Biotechnology Information (NCBI) was NM_000314 and both, the PTEN cDNA and siRNAs, were synthesized by Sangon Biotech. Cells were seeded in six-well plates and transfected with the miR-221 mimic, miR-221 inhibitor or their negative controls using Lipofectamine 3000 transfection reagent (Cat. No. 11668019; Invitrogen, Carlsbad, USA) according to the manufacturer's instructions. Transfections were performed on days 4 and 7. All subsequent experiments were performed $24 \mathrm{~h}$ after the final transfection.

\section{Western blot}

Total protein from the EPCs was extracted using radioimmunoprecipitation assay (RIPA) buffer and then separated using 10\% sodium dodecyl sulfate polyacrylamide gel electrophoresis (SDS-PAGE) before being transferred to polyvinylidene fluoride (PVDF) membranes using electrophoresis, and probed with primary antibodies against $\alpha$ SMA (Cat.
No. CBL171; Merck Millipore, Billerica, USA), myocardin (Cat. No. 14760S; Cell Signaling Technology, Danvers, USA), PTEN (Cat. No. sc-7974; Santa Cruz Biotechnology, Dallas, USA), p-FoxO3a (Cat. No. sc-101683; Santa Cruz Biotechnology), FoxO3a (Cat. No. 2497s, Cell Signaling Technology), and $\beta$-actin (Cat. No. 3700; Cell Signaling Technology). Appropriate horseradish peroxidase (HRP)-conjugated secondary antibodies were then used to facilitate visualization, and protein bands were detected using an electrochemiluminescence (ECL) system (Fusion FX7; Vilber, Lemont, USA).

\section{Statistical analyses}

All data were initially tested using the one-sample Kolmogorov-Smirnov normality test and F-test. This highlighted that not all data were normally distributed and that some data variance was uneven. All experiments were repeated 5 times and therefore we used the more reliable nonparametric tests. The data were expressed as maximum, minimum, median, percentiles 25 , and percentiles 75 , and analyzed with the Kruskal-Wallis $\mathrm{H}$ test followed by a Mann-Whitney U test for post hoc comparison, using the SPSS v. 13.0 software (SPSS Inc., Chicago, USA). The values of $\mathrm{p} \leq 0.05$ were considered statistically significant.

\section{Results}

\section{TGF- $\beta 1$ induces EndMT in EPCs}

Endothelial progenitor cells were identified as described in our previous research. ${ }^{7}$ The cells cultured from mononuclear cells showed a cobblestone-like appearance, while EPCs treated with TGF- $\beta 1$ showed a spindle-shaped appearance (Fig. 1A,B). Immunofluorescent staining showed that EPCs treated with TGF- $\beta 1$ had a high SM $22 \alpha$ expression (Fig. 1C,D) and the expression of $\alpha$ SMA also increased in response to TGF- $\beta 1$ (Fig. 1E).

\section{miR-221 expression was decreased following EPC EndMT}

The miR-221 expression continuously increased in EPCs cultured in medium without TGF- $\beta 1$. In contrast, when

Table 1. The primer sequences used for this investigation

\begin{tabular}{l|c|c|}
\multicolumn{1}{|c|}{ Gene } & Forward $5^{\prime} \rightarrow 3^{\prime}$ & Reverse $^{\prime \prime} \rightarrow 3^{\prime}$ \\
\hline miR-221 & CGCGAGCTACATTGTCTGCTG & AGTGCAGGGTCCGAGGTATT \\
\hline PTEN & TTGAAGACCATAACCCACCACAGC & CATTACACCAGTCCGTCCTTTCCC \\
\hline PIK3R1 & ACTGAAGCAGATGTTGAACAAC & CATCGATCATTTCCAAGTCCAC \\
\hline FOXO3a & TGGACGCCTGGACCGACTTC & GCTCCGTGCTCGCCAAGATG \\
\hline ESR1 & CTGCTGCTGGAGATGCTGGATG & CTGGCTCTGGCTAGGCTCCTC \\
\hline GAPDH 1 & TGTTCGCCTTCTACAGAGGAGACC & CTGGTGGGATGTGTGAGCAAGTC \\
\hline
\end{tabular}



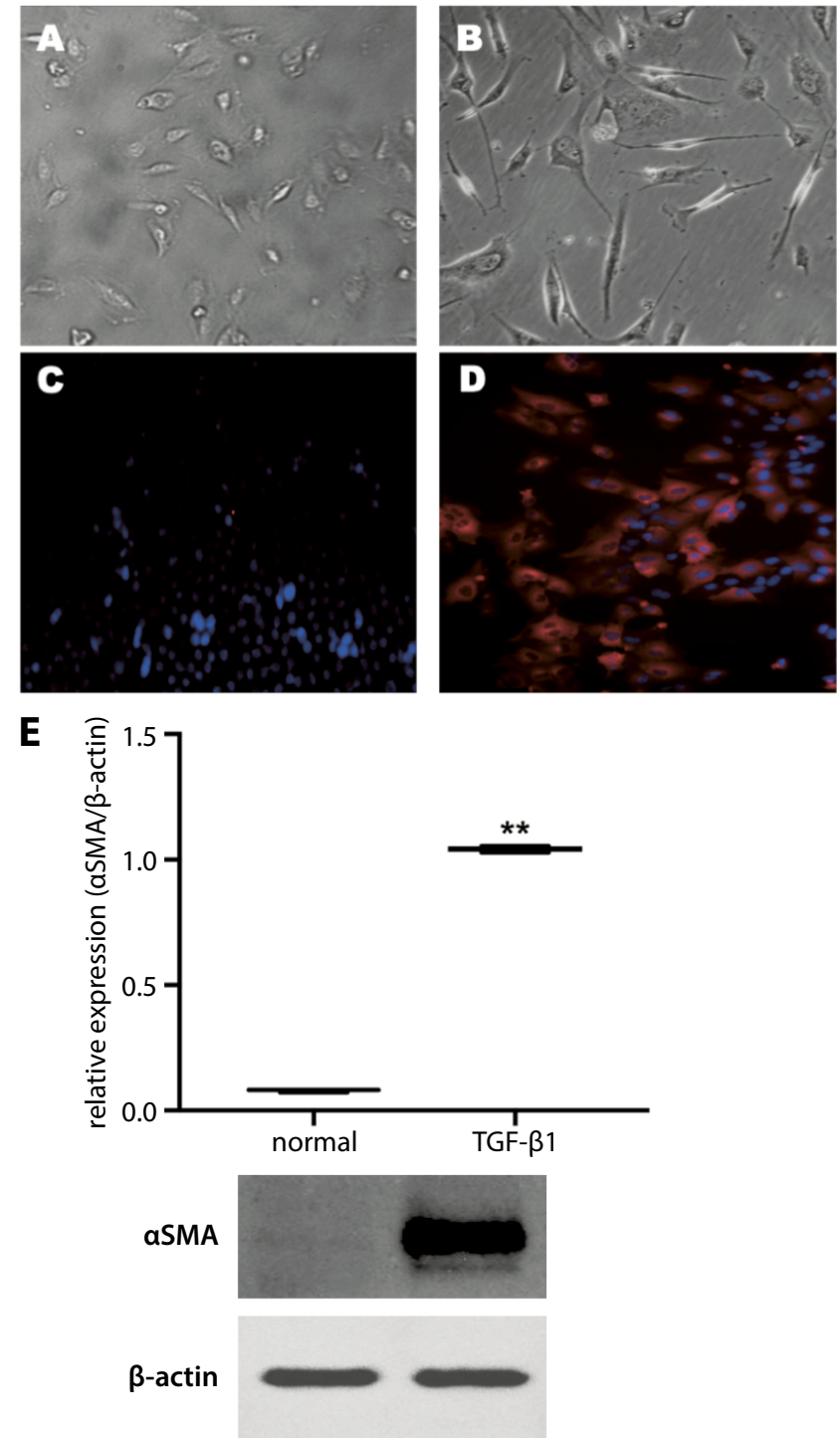

Fig. 1. TGF- $\beta 1$ induces endothelial-mesenchymal transition (EndMT) in endothelial progenitor cells (EPCs). A and B. Morphology of EPCs under a light microscope; A. EPCs cultured in endothelial cell basal medium-2 (EBM-2) containing 20\% fetal bovine serum (FBS); B. The EPCs treated with TGF- $\beta 1$; C and D. Immunofluorescence staining of SM22a in EPCs (red: SM22a; blue: DAPI); C. The EPCs cultured in EBM-2 containing 20\% FBS; $D$. The EPCs treated with TGF- $\beta 1$; E. The expression of aSMA in EPCS with or without TGF- $\beta 1$ was detected with western blot (data expressed as maximum, minimum, median, percentiles 25 and percentiles 75 , and analyzed with Kruskal-Wallis $\mathrm{H}$ test followed by Mann-Whitney $\mathrm{U}$ test for post hoc comparison using the SPSS v. 13.0 software). Normal - EPCS cultured in EBM-2 containing 20\% FBS; TGF $\beta 1$ - EPCs treated with TGF- $\beta 1$; ${ }^{* *} p<0.01$

EPCs were treated with TGF- $\beta 1$, the miR-221 expression remained comparable over the first 3 days and then began to gradually decrease compared to controls (Fig. 2A).

\section{Overexpression of miR-221 inhibits TGF- $\beta 1$-induced EndMT of EPCs}

To demonstrate the role of miR-221 in EPC EndMT, EPCs were transfected with either a miR-221 inhibitor

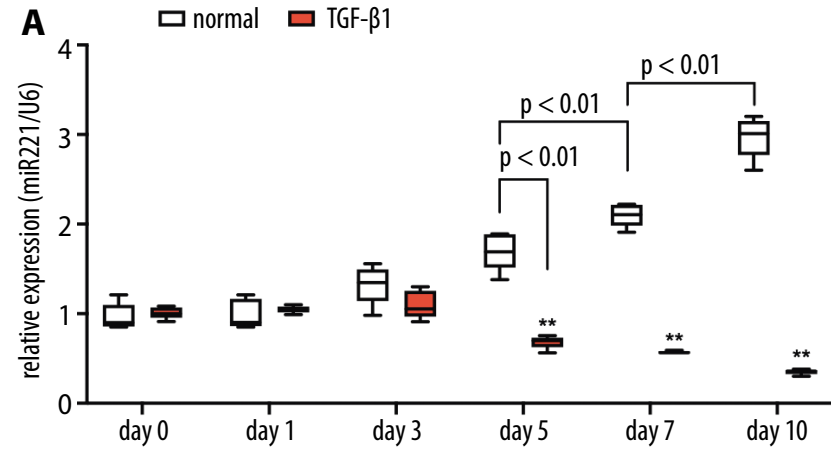
B $\square$ normal $\quad \square$ TGF- $\beta 1+$ miR221 inhibitor control $\square$ TGF- $\beta 1 \quad \square$ miR-221 mimics

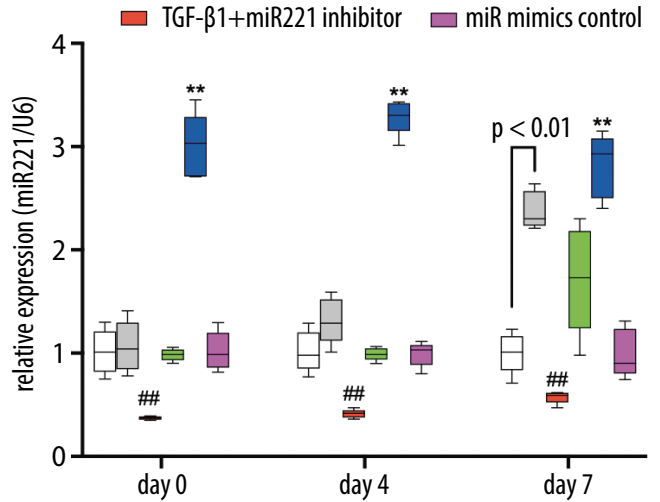

Fig. 2. The expression of miR-221 in endothelial progenitor cells (EPCS) during endothelial-mesenchymal transition (EndMT). A. The expression of miR-211 in EPCs with or without TGF- $\beta 1$ over 10 days and detected with reverse transcription polymerase chain reaction (RT-PCR). Normal - EPCs cultured in endothelial cell basal medium-2 (EBM-2) containing $20 \%$ fetal bovine serum (FBS); TGF $\beta 1$ - EPCs treated with TGF- $\beta 1$; ${ }^{* *}$ normal cpmpared to TGF- $\beta 1, p<0.01$; B. The expression of miR-221 every 3 days following the treatment with miR-221 mimics and inhibitors as detected with RT-PCR. The data were expressed as maximum, minimum, median, percentiles 25 and percentiles 75, and analyzed with Kruskal-Wallis H test, followed by Mann-Whitney $U$ test for post hoc comparison. Normal - EPCs cultured in EBM-2 containing 20\% FBS; TGFB1 - EPCs treated with TGF- $\beta 1$; TGF $\beta 1+$ miR-221 inhibitor - EPCs transfected with miR-22 inhibitor then treated with TGF- $\beta 1$; TGF $1+$ miR-221 inhibitor control - EPCs transfected with the negative control of miR-221 inhibitor then treated with TGF- $\beta 1$; miR-221 mimics - EPCs cultured in EBM-2 containing $20 \%$ FBS and transfected with miR-221 mimics; miR-221 mimics control - EPCs cultured in EBM-2 containing 20\% FBS and transfected with the negative control of miR-221 mimics; ${ }^{* *}$ normal compared to miR-221 mimics, $p<0.01$; \# TGF- $\beta 1$ compared to TGF- $\beta 1+$ miR-221 inhibitor, $p<0.01$

or miR-221 mimic (Fig. 2B), and then treated with TGF- $\beta 1$. Endothelial progenitor cells transfected with the miR-221 inhibitor exhibited a spindle-shaped appearance, while EPCs transfected with miR-221 mimics presented with a cobblestone-like appearance (Fig. 3A-C). Immunofluorescent staining revealed that the SM22 $\alpha$ expression significantly increased or decreased in response to the miR221 inhibitor or miR-221 mimic, respectively (Fig. 3D-G). The expression of both $\alpha$ SMA and myocardin was significantly increased in the presence of the miR-221 inhibitor and attenuated in the presence of miR-221 mimics in EPCs treated with TGF- $\beta 1$ (Fig. 4A,B). 

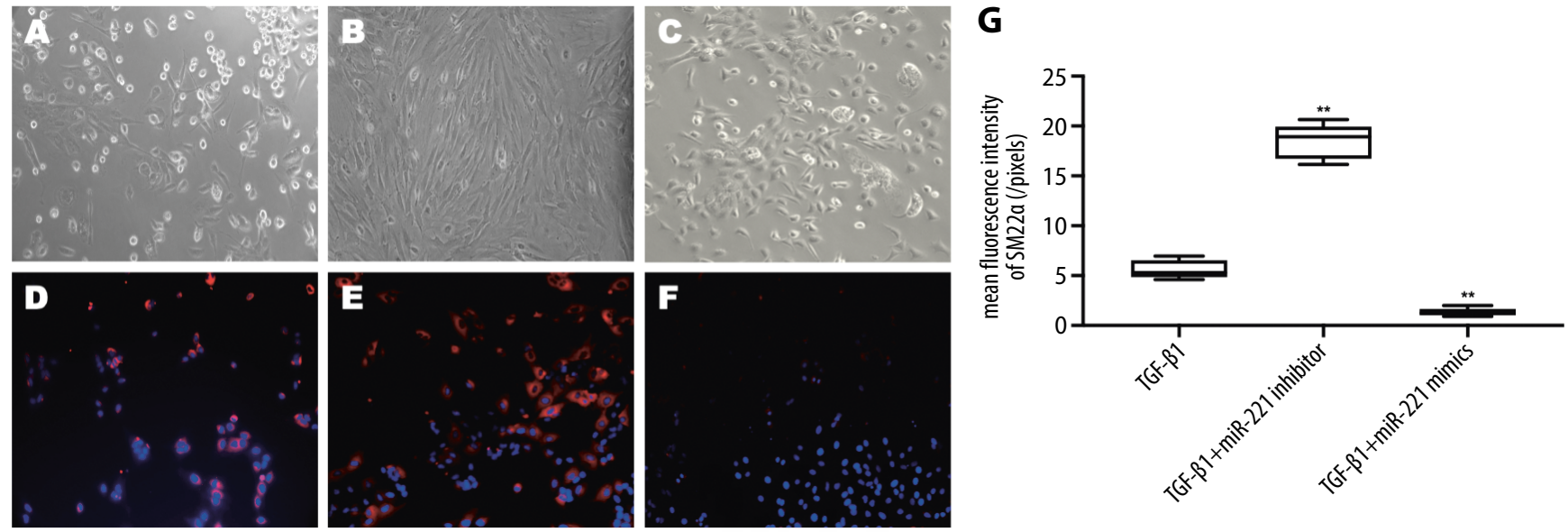

Fig. 3. Morphological and SM22a expression changes in endothelial progenitor cells (EPCs) following miR-221 overexpression or silencing during endothelialmesenchymal transition (EndMT). A-C. Morphological changes in EPCs evaluated using light microscopy; A. The EPCs treated with TGF- $\beta 1$; B. The EPCs transfected with miR-221 inhibitors and treated with TGF- $\beta 1$; C. The EPCs transfected with miR-221 mimics and treated with TGF- $\beta 1$; D-F. Immunofluorescence staining of SM22a in EPCs. The expression of SM22a in EPCs during EndMT following miR-221 overexpression or silencing; D. The EPCs treated with TGF- $\beta 1$; E. Silencing of miR-221 in EPCs treated with TGF- $\beta 1$; F. Overexpression of miR-221 in EPCs treated with TGF- $\beta 1$; G. Mean fluorescence intensity of SM22a in EPCs following miR-221 overexpression or silencing during EndMT. Data expressed as maximum, minimum, median, percentiles 25 and percentiles 75 , and analyzed with Kruskal-Wallis $\mathrm{H}$ test followed by Mann-Whitney $U$ test for post hoc comparison. TGF $\beta 1$ - EPCs treated with TGF- $\beta 1 ;$ TGF $\beta 1+$ miR-221 inhibitor - EPCs transfected with miR-221 inhibitor and treated with TGF- $\beta 1$; TGF $\beta 1$ + miR-221 mimics - EPCs transfected with miR-221 mimics and treated with TGF- $\beta 1$; ${ }^{* *} p<0.01$

A

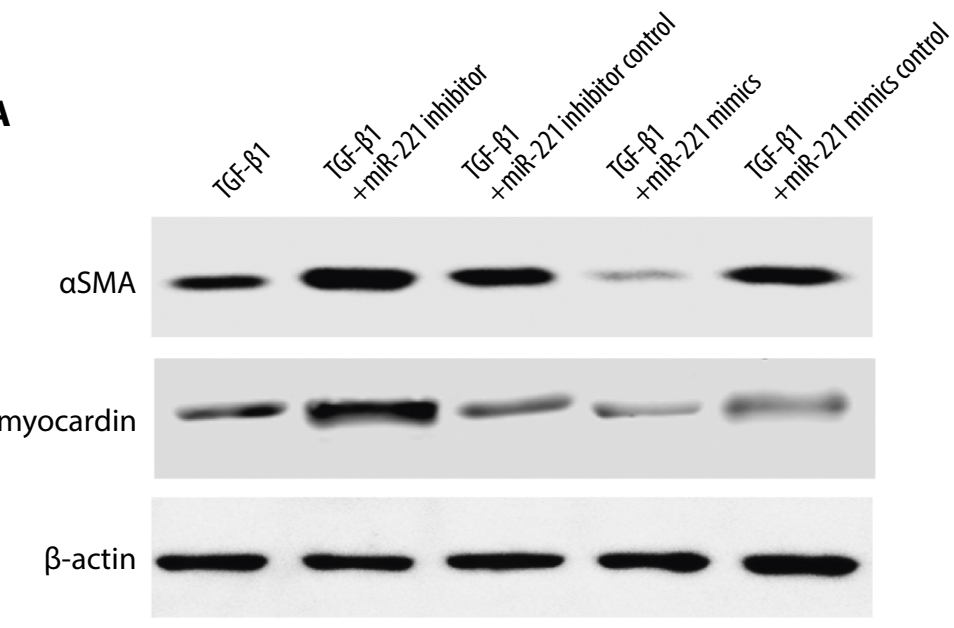

B

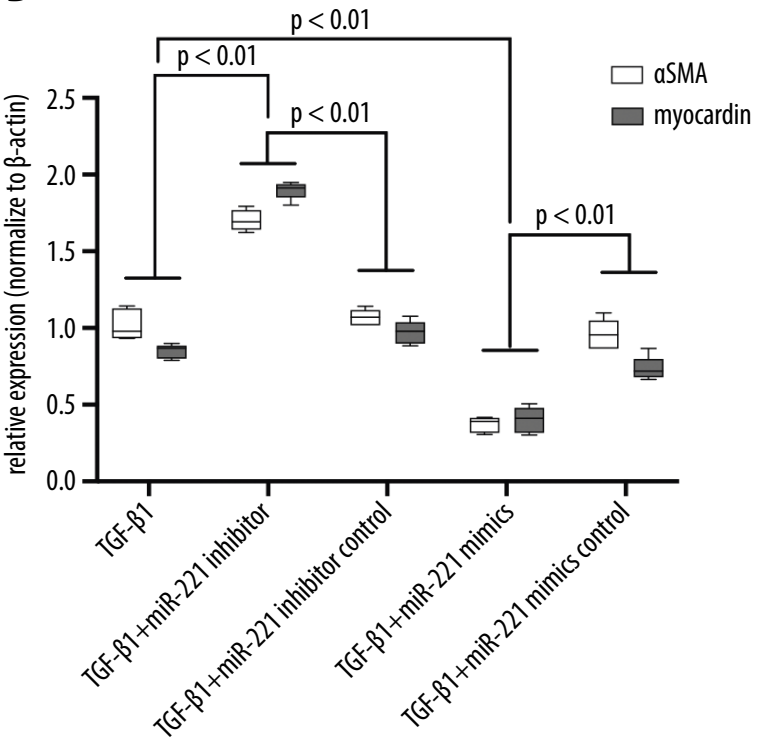

Fig. 4. Expression of aSMA and myocardin in endothelial progenitor cells (EPCs) undergoing endothelial-mesenchymal transition (EndMT). A. Western blot describing aSMA and myocardin expression during EndMT in EPCs; B. The relative expression of aSMA and myocardin under these conditions. Data expressed as maximum, minimum, median, percentiles 25 and percentiles 75, and analyzed with Kruskal-Wallis H test followed by Mann-Whitney $U$ test for post hoc comparison. TGF $\beta 1$ - EPCs treated with TGF- $\beta 1$; TGF $\beta 1$ + miR221 inhibitor - EPCs transfected with miR-221 inhibitor then treated with TGF- $\beta 1$; TGF $\beta 1+$ miR221 inhibitor control - EPCs transfected with the negative control of miR-221 inhibitor and treated with TGF- $\beta 1$; TGF $\beta 1+$ miR221 mimics - EPCs transfected with miR-221 mimics and treated with TGF- $\beta 1$; TGF $\beta 1+$ miR221 mimics control - EPCs transfected with the negative control of miR-221 mimics and treated with TGF- $\beta 1$

miR-221 regulates

\section{the PTEN/FoxO3 signaling pathways} in TGF- $\beta 1$-induced EndMT

The expression of several potential targets of miR-221 in EPCs undergoing TGF- $\beta 1$-dependent EndMT was assessed with RT-PCR. The expression of PTEN was shown to be significantly higher in EPCs treated with TGF- $\beta 1$, while the expression of PIK3R1, FoxO3a, ESR1, and MMP-1 was comparable between the 2 groups (Fig. 5A). The expression of $\alpha$ SMA was increased in TGF- $\beta 1$-treated EPCs and was attenuated in response to PTEN siRNA. However, 


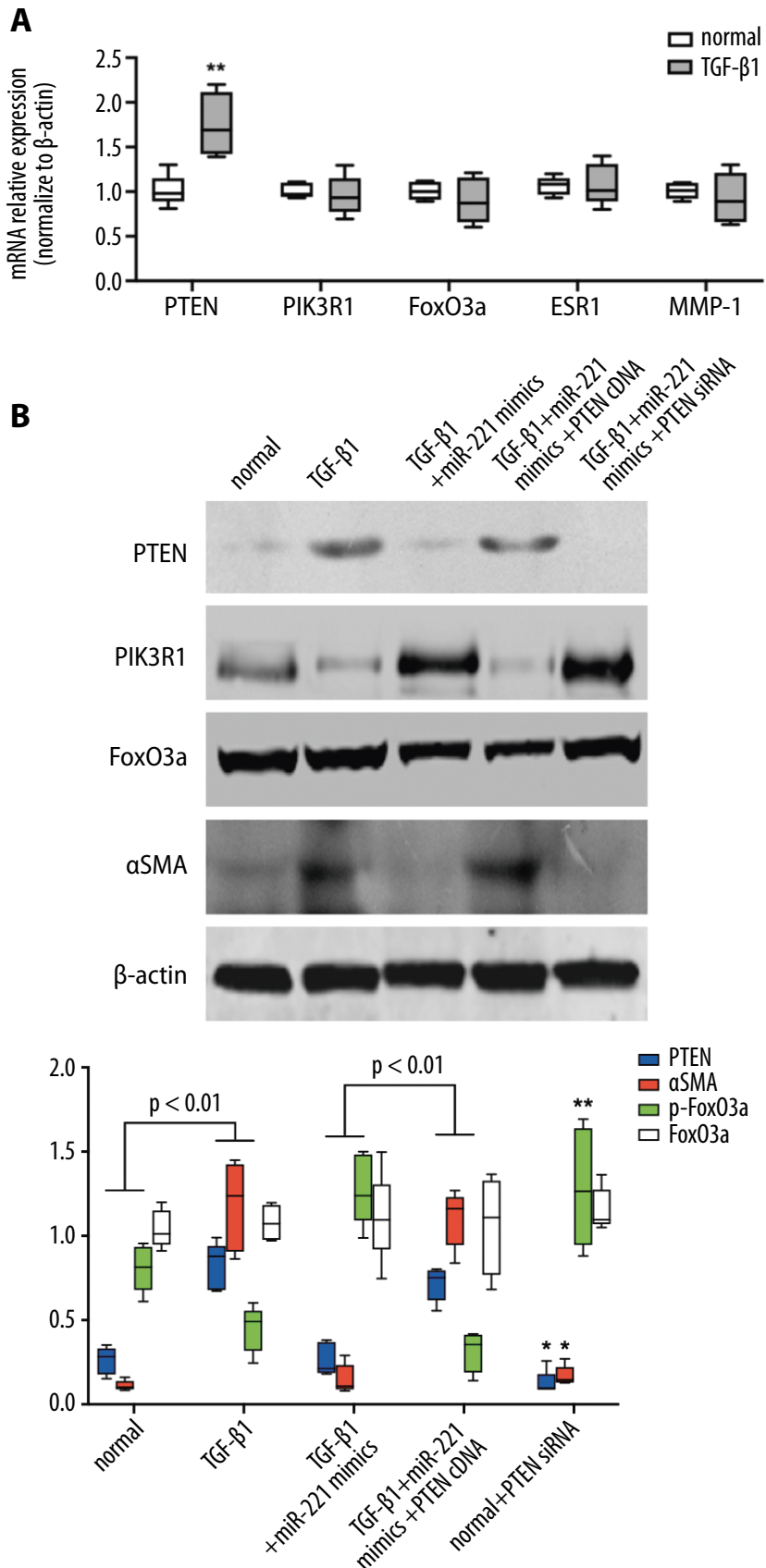

Fig. 5. Expression of miR-221 related genes in endothelial progenitor cells (EPCs) during endothelial-mesenchymal transition (EndMT).

A. The expression of phosphatase and tensin homolog (PTEN), PI3KR1, FoxO3a, ESR1, and MMP-1 during EndMT was detected with reverse transcription polymerase chain reaction (RT-PCR). Normal - EPCs cultured in endothelial cell basal medium-2 (EBM-2) containing 20\% fetal bovine serum (FBS); TGF $\beta 1$ - EPCs treated with TGF- $\beta 1$, ${ }^{* *} p<0.01 ; B$. Western blot evaluating PTEN, p-FoxO3a, FoxO3a, and aSMA expression.

Data expressed as maximum, minimum, median, percentiles 25 and percentiles 75, and analyzed with Kruskal-Wallis H test followed by MannWhitney $U$ test for post hoc comparison. Normal - EPCs cultured in EBM-2 containing 20\% FBS; TGF $\beta 1$ - EPCs treated with TGF- $\beta 1$; TGF $\beta 1+$ miR221 mimics - miR-221 overexpressing EPCs treated with TGF- $\beta 1$; TGF $\beta 1+$ miR221 mimics + PTEN cDNA - miR-221 and PTEN overexpressing EPCs treated with TGF- $\beta 1$; normal + PTEN siRNA - PTEN silenced EPCs cultured in EBM-2 containing 20\% FBS; ${ }^{*}$ normal compared to normal + PTEN siRNA 5, $p<0.05 ;{ }^{* *}$ normal compared to normal + PTEN siRNA, $p<0.01$ the increase in $\alpha$ SMA expression was significantly attenuated by the addition of miR-221 mimics in EPCs treated with TGF- $\beta 1$, which was reversed in response to PTEN cDNA (Fig. 5B). At the same time, the total expression of FoxO3a remained unchanged. However, phosphorylated FoxO3a expression was notably reduced in response to TGF- $\beta 1$, which was reversed following the addition of the miR-221 mimics. This reversal was nearly completely abolished following the addition of the PTEN cDNA. In addition, the phosphorylation of FoxO3a was significantly augmented in EPCs treated with PTEN siRNA compared to normal cells (Fig. 5B).

\section{Discussion}

It has been widely reported that EPCs are beneficial in the treatment of coronary heart disease, as these cells can differentiate into mature vascular endothelial cells. However, accumulating evidence suggests that EPCs can also differentiate into mesenchymal cells, which may aggravate intimal hyperplasia and contribute to atherogenesis. ${ }^{18,19}$ Several studies have shown that TGF- $\beta 1$ induces the EndMT process in EPCs in vitro. However, only half of the EPCs transplanted into the impaired endothelium were transformed into smooth muscle cells (SMCs) following TGF- $\beta 1$ treatment, while the others acted to enhance intimal hyperplasia in vivo. ${ }^{2}$ These results suggest that the EndMT process in EPCs can be regulated. In this study, we found that a deficiency in miR-221 may facilitate TGF- $31-$ dependent EndMT in EPCs. Furthermore, miR221 inhibits the EndMT process by interacting with PTEN, which regulates PTEN/FoxO3 signaling.

The SM22 $\alpha$ and $\alpha$ SMA are mesenchymal markers used to detect EndMT in EPCs, as described previously. ${ }^{7}$ In this study, the expression of SM $22 \alpha$ and $\alpha$ SMA significantly increased in EPCs after the treatment with TGF- $\beta 1$, suggesting that EPCs underwent EndMT. ${ }^{7}$ It has also been reported that EPCs derived from rat bone marrow are able to undergo EndMT after treatment with TGF- $\beta 1,{ }^{2}$ while human circulating EPCs only differentiated into mesenchymal cells following direct contact with smooth muscle cells, ${ }^{19}$ indicating a complex regulatory system in different tissues.

Some studies have reported that miRs are closely associated with cellular transdifferentiation. The miRNA-221 exerts anti-angiogenic, anti-inflammatory and pro-apoptotic effects in EPCs through their interactions with ZEB2 and Ets-1. ${ }^{10,11}$ In ectopic endometrial tissues and ectopic endometrial stromal cells, the inhibition of miR-221 expression leads to decreased cellular proliferation. ${ }^{20}$ In our study, we found that the expression of miR-221 was markedly decreased for up to 5 days following TGF- $\beta 1$ treatment, and was accompanied by an increase in $\alpha$ SMA expression. This suggests that miR-221 is involved in the EndMT process of EPCs. 
To determine the role of miR-221 in EndMT, miR-221 inhibitor or mimics were repeatedly transfected into EPCs following TGF- $\beta 1$ treatment on days 1,4 and 7 , to induce either upregulation or downregulation of miR-221. As a result, the expression of SM22 $\alpha$, $\alpha$ SMA and myocardin in EPCs undergoing EndMT was either increased or decreased following transfection with miR-221 inhibitors and miR-221 mimics, respectively. The previous study suggests that myocardin plays a critical role in the development of cardiac myocytes and vascular SMCs. Loss of myocardin results in a synthetic, dedifferentiated phenotype that leads to atherosclerosis. ${ }^{21}$ These results suggest that miR221 inhibits the EndMT process in EPCs by suppressing the expression of $\alpha \mathrm{SMA}$ and myocardin.

Previous studies have also reported that the PI3K/ Akt/FoxO3 signaling pathway plays an important role in the self-renewal of hematopoietic stem cells. ${ }^{22}$ In our previous study, we also found that miR-126 targets PI3KR2 to inhibit EPC EndMT through the PI3K/Akt/FoxO3 pathway. ${ }^{7}$ In this study, we evaluated several potential target genes for miR-221, including PTEN, PI3KR1, FoxO3a, ESR1, and $M M P-1$, in EPCs undergoing EndMT. We found that the expression of PTEN was increased in EPCs treated with TGF- $\beta 1$, while its expression was inhibited by overexpression of miR-221. The expression levels of PI3KR2, FoxO3a, ESR1, and $M M P-1$ were comparable between the 2 groups. These results suggest that PTEN might be a target of miR221 and regulate the EndMT process in EPCs. This was supported by the finding that miR-221 overexpression amplified PI3K signaling to promote precursor B-cell retention by targeting PTEN. ${ }^{23}$

In addition, we found that FoxO3a expression was slightly decreased in EPCs treated with TGF- $\beta 1$. A downstream effector of the PTEN/Akt pathway, FoxO3a, is localized within the nucleus, where it drives transcription. However, when phosphorylated, the protein translocates to the cytoplasm. ${ }^{24}$ One example of this kind of regulation can be seen in the differential regulation of onco- and anti-oncogene expression in cells following the nuclear translocation of phosphorylated AKT. ${ }^{14,25}$ Interestingly, we found that the expression of phosphorylated FoxO3a (p-FoxO3a) was significantly increased when PTEN was suppressed, while overexpression of PTEN led to the inhibition of p-FoxO3a expression. This further suggests that PTEN decreases the phosphorylation of FoxO3a, thereby suppressing its nuclear translocation in EPCs undergoing TGF- $\beta 1$-dependent EndMT. Furthermore, we found that miR-221 overexpression led to a decrease in PTEN expression and an increase in $\alpha \mathrm{SMA}$ expression, both of which were reversed by PTEN overexpression. Finally, our previous study demonstrated that the phosphorylation of FoxO3a negatively regulates EPC EndMT. ${ }^{7}$ Taken together, these findings suggest that miR-221 may lead to PTEN downregulation and the activation of the FoxO3a signaling pathway, thereby inhibiting the EndMT process in EPCs.

\section{Limitations}

In the present study, we report that the overexpression of miR-221 inhibits EndMT in EPCs, but as all experiments in this study were conducted in vitro, there should be some focus on validating these findings in vivo. We believe that miR-221 interacts with PTEN to regulate FoxO3a/Smad4 transcription, but we did not evaluate Smad4 expression in this study, as these observations were recorded in our previous paper. ${ }^{7}$ Our findings suggest that miR-221 may be a potential target during the treatment of in-stent restenosis in coronary artery disease, and facilitate the inhibition of intimal hyperplasia through EndMT signaling.

\section{Conclusions}

In summary, we found that the overexpression of miR221 inhibited EndMT in EPCs. This phenomenon is likely the result of the interaction between miR-221 and its target gene, $P T E N$, effecting a change in the regulation of FoxO3a phosphorylation.

\section{ORCID iDs}

En Zhou (1) https://orcid.org/0000-0002-6073-8037

Yinghua Zou (1) https://orcid.org/0000-0002-6596-8720

Chengyu Mao (1) https://orcid.org/0000-0001-9740-8835

Dongjiu Li (10) https://orcid.org/0000-0003-4372-6613

Changqian Wang (1) https://orcid.org/0000-0002-7611-7761

Zongqi Zhang (1) https://orcid.org/0000-0001-6785-2038

\section{References}

1. Toya SP, Malik AB. Role of endothelial injury in disease mechanisms and contribution of progenitor cells in mediating endothelial repair. Immunobiology. 2012;217(5):569-580. doi:10.1016/j.imbio.2011.03.006

2. Imamura H, Ohta T, Tsunetoshi K, et al. Transdifferentiation of bone marrow-derived endothelial progenitor cells into the smooth muscle cell lineage mediated by tansforming growth factor-beta1. Atherosclerosis. 2010;211(1):114-121. doi:10.1016/j.atherosclerosis. 2010.02.040

3. Hong L, Du X, Li W, Mao Y, Sun L, Li X. EndMT: A promising and controversial field. Eur J Cell Biol. 2018;97(7):493-500. doi:10.1016/j.ejcb. 2018.07.005

4. Peinado VI, Ramírez J, Roca J, Rodriguez-Roisin R, Barberà JA. Identification of vascular progenitor cells in pulmonary arteries of patients with chronic obstructive pulmonary disease. Am J Respir Cell Mol Biol. 2006;34(3):257-263. doi:10.1165/rcmb.2005-0255OC

5. Lee $\mathrm{Y}, \mathrm{Kim} \mathrm{M}$, Han J, et al. MicroRNA genes are transcribed by RNA polymerase II.EMBO J. 2004;23(20):4051-4060. doi:10.1038/sj.emboj. 7600385

6. Cai $\mathrm{X}$, Hagedorn $\mathrm{CH}$, Cullen BR. Human microRNAs are processed from capped, polyadenylated transcripts that can also function as mRNAs. RNA. 2004;10(12):1957-1966. doi:10.1261/rna.7135204

7. Zhang J, Zhang Z, Zhang DY, Zhu J, Zhang T, Wang C. microRNA 126 inhibits the transition of endothelial progenitor cells to mesenchymal cells via the PIK3R2-PI3K/Akt signalling pathway. PloS One. 2013;8(12):e83294. doi:10.1371/journal.pone.0083294

8. Kumarswamy R, Volkmann I, Jazbutyte V, Dangwal S, Park DH, Thum T. Transforming growth factor- $\beta$-induced endothelial-to-mesenchymal transition is partly mediated by microRNA-21. Arterioscler Thromb Vasc Biol. 2012;32(2):361-369. doi:10.1161/atvbaha.111.234286

9. Ghosh AK, Nagpal V, Covington JW, Michaels MA, Vaughan DE. Molecular basis of cardiac endothelial-to-mesenchymal transition (EndMT): Differential expression of microRNAs during EndMT. Cell Signal. 2012;24(5):1031-1036. doi:10.1016/j.cellsig.2011.12.024 
10. Chen Y, Banda M, Speyer CL, Smith JS, Rabson AB, Gorski DH. Regulation of the expression and activity of the antiangiogenic homeobox gene GAX/MEOX2 by ZEB2 and microRNA-221. Mol Cell Biol. 2010; 30(15):3902-3913. doi:10.1128/mcb.01237-09

11. Zhu N, Zhang D, Chen S, et al. Endothelial enriched microRNAs regulate angiotensin II-induced endothelial inflammation and migration. Atherosclerosis. 2011;215(2):286-293. doi:10.1016/j.atherosclerosis. 2010.12.024

12. Ni HZ, Liu Z, Sun LL, et al. Metformin inhibits angiogenesis of endothelial progenitor cells via miR-221-mediated p27 expression and autophagy. Future Med Chem. 2019;11(17):2263-2272. doi:10.4155/ fmc-2019-0017

13. Peng $H$, Yang $H$, Xiang $X$, Li S. MicroRNA-221 participates in cerebral ischemic stroke by modulating endothelial cell function by regulating the PTEN/PI3K/AKT pathway. Exp Ther Med. 2020;19(1):443-450. doi:10.3892/etm.2019.8263

14. Zhang Q, Li X, Li Y, et al. Expression of the PTEN/FOXO3a/PLZF signalling pathway in pancreatic cancer and its significance in tumourigenesis and progression. Invest New Drugs. 2020;38(2):321-328. doi:10. 1007/s10637-019-00791-7

15. Mziaut H, Henniger G, Ganss K, et al. MiR-132 controls pancreatic beta cell proliferation and survival through Pten/Akt/Foxo3 signaling. Mol Metab. 2020;31:150-162. doi:10.1016/j.molmet.2019.11.012

16. Zhang Z, Zhang T, Zhou Y, et al. Activated phosphatidylinositol 3-kinase/Akt inhibits the transition of endothelial progenitor cells to mesenchymal cells by regulating the forkhead box subgroup $0-3 a$ signaling. Cell Physiol Biochem. 2015;35(4):1643-1653. doi:10.1159/ 000373978

17. Moonen JR, Krenning G, Brinker MG, Koerts JA, van Luyn MJ, Harmsen MC Endothelial progenitor cells give rise to pro-angiogenic smooth muscle-like progeny. Cardiovasc Res. 2010;86(3):506-515. doi:10.1093/ cvr/cvq012
18. Rotmans Jl, Heyligers JM, Verhagen HJ, et al. In vivo cell seeding with anti-CD34 antibodies successfully accelerates endothelialization but stimulates intimal hyperplasia in porcine arteriovenous expanded polytetrafluoroethylene grafts. Circulation. 2005;112(1): 12-18. doi:10.1161/CIRCULATIONAHA.104.504407

19. Diez M, Musri MM, Ferrer E, Barbera JA, Peinado VI. Endothelial progenitor cells undergo an endothelial-to-mesenchymal transition-like process mediated by TGFbetaRI. Cardiovasc Res. 2010;88(3):502-511. doi:10.1093/cvr/cvq236

20. Du XH, Shi G, Lü DH, et al. The expression of microRNA-221 in endometriosis and its impact on endometrial stromal cells [in Chinese]. Sichuan DaXue Xue Bao YiXue Ban. 2018;49(4):546-550.PMID:30378307

21. Xia XD, Zhou Z, Yu XH, Zheng XL, Tang CK. Myocardin: A novel player in atherosclerosis. Atherosclerosis. 2017;257:266-278. doi:10.1016/j. atherosclerosis.2016.12.002

22. Miyamoto K, Araki KY, Naka K, et al. Foxo3a is essential for maintenance of the hematopoietic stem cell pool. Cell Stem Cell. 2007;1(1): 101-112. doi:10.1016/j.stem.2007.02.001

23. Petkau G, Kawano Y, Wolf I, Knoll M, Melchers F. MiR221 promotes precursor B-cell retention in the bone marrow by amplifying the PI3Ksignaling pathway in mice. Eur J Immunol. 2018;48(6):975-989. doi:10. 1002/eji.201747354

24. Wang K, Li PF. Foxo3a regulates apoptosis by negatively targeting miR-21. J Biol Chem. 2010;285(22):16958-16966. doi:10.1074/jbc.M109. 093005

25. Jang $\mathrm{H}$, Lee $\mathrm{OH}$, Lee $\mathrm{Y}$, et al. Melatonin prevents cisplatin-induced primordial follicle loss via suppression of PTEN/AKT/FOXO3a pathway activation in the mouse ovary. J Pineal Res. 2016;60(3):336-347. doi:10.1111/jpi.12316 